\title{
The causes of serous macular detachment excluding central serous chorioretinopathy
}

This article was published in the following Dove Press journal:

Clinical Optometry

28 May 2010

Number of times this article has been viewed

\section{Burak Turgut \\ Fatih Cem Gul \\ Tamer Demir \\ Ulku Celiker}

Department of Ophthalmology, Fırat University School of Medicine, Elazığ, Turkey
Correspondence: Burak Turgut Firat University School of Medicine, Department of Ophthalmology, Elazığ, Turkey

Tel +904242333555

Fax +90424238 8096

Email drburakturgut@gmail.com
Objective: In our study, we investigate the causes of serous macular detachment (SMD) excluding central serous chorioretinopathy (CSC).

Study design/patients and methods: A retrospective observational study. The medical records of 58 patients with SMD documented by optical coherence tomography (OCT) were reviewed retrospectively. The patients with CSC were excluded from the study. Ocular diseases accompanied with SMD, unilaterality or bilaterality, and demographical properties of the patients were evaluated.

Results: Of the 58 patients with SMD in this study, 20 (34.4\%) were women and 38 (65.5\%) were men. The ages of patients were between 17 to 81 years (median 61.01 years). The causes of SMD were as follow: diabetic macular edema (DME) in 37 patients $(63.8 \%)$, age-related macular degeneration (AMD) associated with choroidal neovascularization in 10 patients (17.24\%), branch retinal vein occlusion in five patients $(8.62 \%)$, central retinal vein occlusion in four patients $(6.90 \%)$, Harada disease in one patient $(1.72 \%)$ and Coat's disease in one patient (1.72\%). Eighteen patients with DME, two patients had AMD associated with choroidal neovascularization, and one patient with Harada disease had bilateral SMD. The most common cause of SMD in both eyes was DME.

Conclusions: The most common cause of SMD excluding CSC seems to be DME. The presence of SMD in retinal vascular diseases such as diabetes might affect the results of the treatment and the prognosis.

Keywords: serous macular detachment, causes, diabetic macular edema, age-related macular degeneration, excluding central serous chorioretinopathy

\section{Introduction}

Serous macular detachment (SMD) may occur in conditions where retinal vascular leakage or retinal pigment epithelium (RPE) dysfunction is seen in disorders such as diabetic macular edema (DME), branch retinal vein occlusion (BRVO), central retinal vein occlusion (CRVO), hypotony maculopathy, retinal vasculitis, and retinal macroaneurysm. ${ }^{1-6}$ Using optical coherence tomography (OCT), Otani et al have described three patterns of DME: sponge-like swelling, cystoid macular edema (CME), and SMD. ${ }^{7}$ Because it may hide beneath DME during fundus fluorescein angiography (FFA), SMD associated with DME can only be diagnosed using OCT.7,8

Although the exact mechanism of development of SMD is not known, it is probably due to excessive fluid flow from the abnormal retinal vessels, which overwhelms the RPE pump leading to serous retinal detachment. ${ }^{1,5,8,9}$ 
The presence of SMD in retinal vascular diseases with retinal vascular leakage may limit the ability to perform effective macular laser treatment. Ohashi et al reported that in ME-associated BRVO, the presence of SMD is a negative prognostic factor for resolution of $\mathrm{ME}$ and for visual acuity (VA) after grid macular laser treatment. ${ }^{10}$ The presence of SMD might be also a prognostic factor in treatment of DME. $^{10-13}$

In the present study, we investigated the causes of SMD excluding central serous chorioretinopathy.

\section{Materials and methods}

In this retrospective study, 84 eyes of 58 patients with SMD were examined between May 2007 and May 2009. The patients with epiretinal membrane or vitreomacular traction documented by OCT, and media opacities such as corneal opacity, lens opacity, vitreous and preretinal hemorrhage, uveitis, proliferative diabetic retinopathy, and patients with history of previous intraocular surgery, macular laser photocoagulation, and intravitreal injection were excluded from the study.

The patients underwent complete ophthalmic examination, including best-corrected VA measurement using the Early Treatment Diabetic Retinopathy Study chart, slitlamp biomicroscopy with a +90 diopter noncontact lens and a Goldmann three-mirror contact lens, FFA, and OCT. The OCT and FFA examinations were performed on the same day, and were evaluated by same. FFA was performed with a retinal camera (Topcon 50IX; Itabashiku, Tokyo, Japan). OCT examinations were performed using spectral OCT and scanning laser ophthalmoscope (SLO) (OCT/SLO; OTI/ OPKO Inc, Toronto, Canada). During OCT examination the maculae were scanned on six radial sections including the horizontal, vertical, and oblique planes through the centre of the fovea. Fluorescein angiographic cystoid, focal, and diffuse ME were thought to be present if typical petaloid hyperfluorescent cystoid spaces radiating from the fovea, focal, and diffuse intraretinal hyperfluorescent spaces were evident during FFA, respectively. OCT examination was thought to show DME if there were hyporeflective intraretinal cavities and/or separated by septa and radiating from the centre of the macula in cross-sectional scans. Angiography could not detect SMD in any case.

Serous macular detachment was thought to be present if the posterior surface of the retina was elevated over a nonreflective black cavity and under ME, with minimal shadowing of the underlying tissues (Figure 1).

The OCT and FFA were performed following the tenets of the Declaration of Helsinki. The study was designed as an institutional, retrospective trial. Informed consents were obtained from the patients.

\section{Results}

Of the 58 patients with SMD in this study, 20 (34.4\%) were women and $38(65.5 \%)$ were men. The ages of patients ranged from 17 to 81 years (median 61.01 years).

We detected six different causes in 58 eyes for SMD. Thirty-seven patients had DME (63.8\%), 10 patients had age-related macular degeneration (AMD) associated with choroidal neovascularization (17.24\%), five patients had

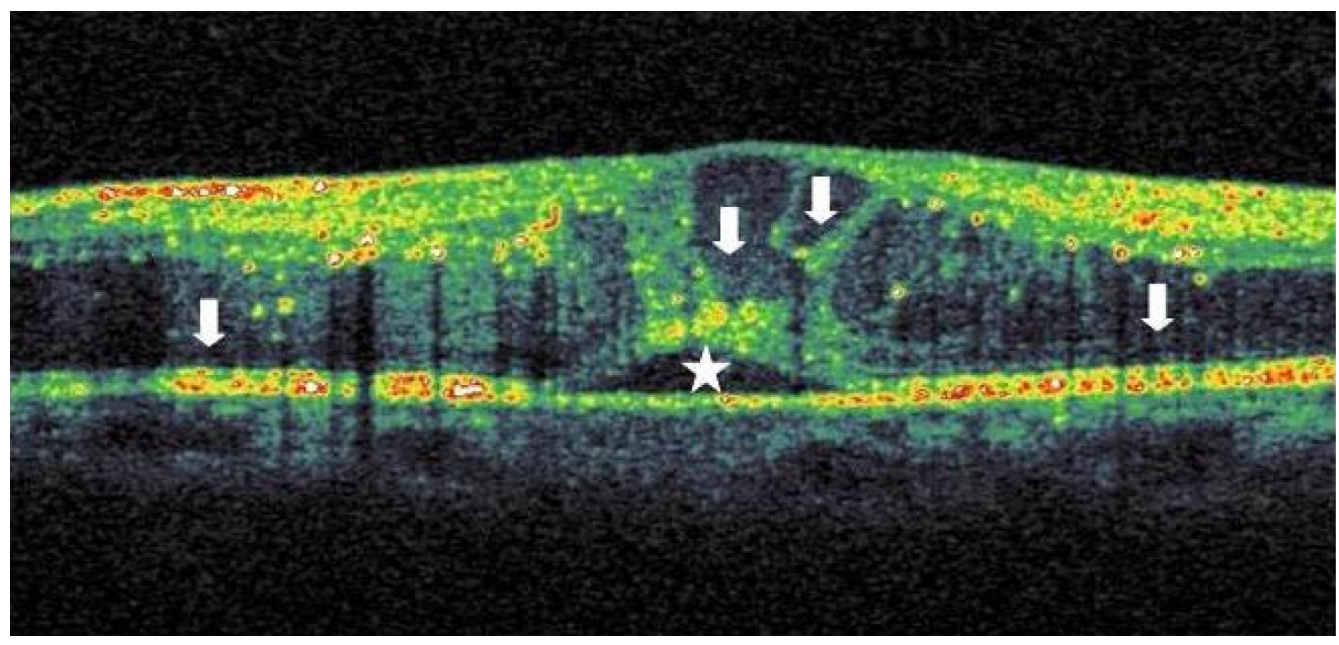

Figure I Serous macula detachment (star) and macular edema (arrows) in a patient with diabetic retinopathy. 
BRVO (8.62\%), four patients had CRVO (6.90\%), one patient had Harada disease $(1.72 \%)$, and one patient had Coat's disease $(1.72 \%)$.

Eighteen patients with DME, two patients with AMD associated with choroidal neovascularization and one patient with Harada disease had bilateral SMD. The most common cause was DME in bilateral cases.

\section{Discussion}

The pathogenesis of SMD associated with retinal vascular disease is not well defined. However, the leakage from retinal or choroidal circulation into the subretinal space that exceeds its compensatory removal capacity is thought to be the main mechanism. SMD has been suggested to be linked not only to the limitations of the draining vascular system, but also to an impairment in the function of the RPE. ${ }^{1-6}$ Kang et al reported that in diabetic eyes, the incidence of CME and SMD increases with the existence of retinal vascular hyperpermeability, and suggested that the pathology of these entities might share a common pathogenic mechanism. ${ }^{9}$

In the healthy retina, the RPE pump and the tight junctions between adjacent RPE cells maintain the dryness of the subretinal space and the attachment of the sensorial retina to the RPE. The breakdown of the RPE pump or the disruption of the tight junctions between adjacent RPE cells results in intraretinal edema and SMD. ${ }^{1-6}$ The presence of SMD is also an important finding in terms of showing defective RPE cells.

Serous macular detachment associated with DME was first defined by Otani et al. ${ }^{7}$ Although they reported that the frequency of SMD in DME was approximately $15 \%$, Ozdemir et al demonstrated with OCT that $31 \%$ of the patients with diabetic DME have SMD and that the prevalence of SMD was much higher than previously reported. ${ }^{7,11}$

Using OCT in the patients with BRVO, Spaide et al found that the prevalence of SMD was $43 \%$ while Takahashi et al reported that $20 \%$ of the cases had SMD. ${ }^{3,12}$

Additionally, Ozdemir et al reported that SMD was found in $82 \%$ of eyes with CRVO. ${ }^{6}$

We found that the prevalence of SMD in this study was higher than previously reported. This may be due to the detection of the SMD using OCT.

We consider that the detection of SMD using OCT in patients with various retinal vascular diseases might be evidence for RPE dysfunction. The presence of SMD in these patients may be important predictors for the success of the treatment interventions, and for prognosis of these diseases.
In this regard, Shukla et al have reported that SMD might be a predictor for the resolution of DME following the injection of intravitreal triamcinolone acetate. ${ }^{13}$ Additionally, Ohashi et al observed that the presence of SMD is a negative prognostic indicator for resolution of ME and for visual acuity after grid macular laser treatment in patients with secondary ME to BRVO. ${ }^{10}$

The size and localization of SMD, the effect of the treatment on the resolution of SMD, and the effect of the presence of SMD on the prognosis in various retinal vascular diseases were not studied in this study. These may be limitations of the study.

In conclusion, the detection of the SMD prevalence in retinal vascular diseases will be easy using OCT. The presence of SMD may significantly limit the ability to perform effective treatments such as macular laser interventions and intravitreal injections. Thus, the detection of SMD by OCT may help guide the treatment of retinal diseases with retinal vascular leaking. Further studies concerning whether the OCT findings correlate with different causes, visual outcome, and prognosis in diseases with SMD may be beneficial for understanding the pathogenesis and prognosis of the disease.

\section{Acknowledgments}

Those involved in conducting the study would like to thank those who assisted with the collection of data, typing, editing, preparation for review, and approval of the final manuscript.

\section{Disclosure}

The authors report no conflicts of interest in this work.

\section{References}

1. Spaide RF, Goldbaum M, Wong DW, Tang KC, Iida T. Serous detachment of the retina. Retina. 2003;23:820-846.

2. Kokame GT, de Leon MD, Tanji T. Serous retinal detachment and cystoid macular edema in hypotony maculopathy. Am J Ophthalmol. 2001;131:384-386.

3. Spaide RF, Lee JK, Klancnik JK Jr, Gross NE. Optical coherence tomography of branch retinal vein occlusion. Retina. 2003;23:343-347.

4. Takahashi K, Kishi S. Serous macular detachment associated with retinal arterial macroaneurysm. Jpn J Ophthalmol. 2006;50:460-464.

5. Hikichi T, Ohtsuka H, Higuchi M, et al. Causes of macular serous retinal detachments in Japanese patients 40 years and older. Retina. 2009;29:395-404.

6. Ozdemir H, Karacorlu M, Karacorlu S. Serous macular detachment in central retinal vein occlusion. Retina. 2005;25:561-563.

7. Otani T, Kishi S, Maruyama Y. Patterns of diabetic macular edema with optical coherence tomography. Am J Ophthalmol. 1999;127:688-693.

8. Kim BY, Smith SD, Kaiser PK. Optical coherence tomographic patterns of diabetic macular edema. Am J Ophthalmol. 2006;142:405-412.

9. Kang SW, Park CY, Ham D. The correlation between fluorescein angiographic and optical coherence tomographic features in clinically significant diabetic macular oedema. Am J Ophthalmol. 2004;137:313-322. 
10. Ohashi H, Oh H, Nishiwaki H, Nonaka A, Takagi H. Delayed absorption of macular edema accompanying serous retinal detachment after grid laser treatment in patients with branch retinal vein occlusion. Ophthalmol. 2004;111:2050-2056.

11. Ozdemir H, Karacorlu M, Karacorlu S. Serous macular detachment in diabetic cystoid macular oedema. Acta Ophthalmol Scand. 2005;83:63-66.
12. Takahashi K, Kashima T, Kishi S. Serous macular detachment combined with branch retinal vein occlusion. Nippon Ganka Gakkai Zasshi. 2005;109:362-367.

13. Shukla D, Behera UC, Chakraborty S, Mahalakshmi R, Prasad NM. Serous macular detachment as a predictor of resolution of macular edema with intravitreal triamcinolone injection. Ophthalmic Surg Lasers Imaging. 2009;40:115-119.

\section{Publish your work in this journal}

Clinical Optometry is an international, peer-reviewed, open access journal publishing original research, basic science, clinical and epidemiological studies, reviews and evaluations on clinical optometry. All aspects of patient care are addressed within the journal as well as the practice of optometry including economic and business analyses. Basic and clinical

\section{Dovepress}

research papers are published that cover all aspects of optics, refraction and its application to the theory and practice of optometry. The manuscript management system is completely online and includes a very quick and fair peer-review system, which is all easy to use. Visit http://www.dovepress. com/testimonials.php to read real quotes from published authors. 